where $\omega$ is the Mach angle for $M=5^{1 / 2}$ and $a, b$ are arbitrary functions of $\alpha, \beta$, respectively. The curves (4.11), (4.12) are logarithmic spirals in the hodograph plane. For the case $v_{0}+q^{2}=0$, the $(x, y)$-plane map of the bicharacteristics $\alpha=$ constant (or $\beta=$ constant), is orthogonal to the $(u, v)$-plane map of $\beta=$ constant (or $\alpha=$ constant). Hence, the (x,y)-plane map of (4.11), (4.12) consists of logarithmic spirals. Specific formulas can be obtained easily. Since the bicharacteristics are inclined to the stream lines at the constant angle $\omega$, the stream lines are also logarithmic spirals. Evidently, a similar method can be used to investigate those degenerate non-steady flows for which $v_{0}=f\left(q^{2}\right), f\left(q^{2}\right)<0$.

\title{
NOTE ON THE CHARACTERISTICS IN UNSTEADY ONE-DIMENSIONAL FLOWS WITH HEAT ADDITION*
}

\author{
By C. C. LIN (Massachusetts Institute of Technology)
}

1. In a very interesting paper, Kahane and Lees ${ }^{1}$ studied the problem of onedimensional wave propagation in a gas when heat is being added. They used the method of numerical integration, by taking finite differences along the characteristics. However, since the form of the characteristic equations contained more than two dependent variables, they were led to use some rather artificial approximations besides those involved in taking finite differences. As the type of work done by Kahane and Lees will probably be continued by people interested in jet propulsion, it seems desirable that the most convenient form of the characteristic equations be derived and a simple procedure of numerical integration be developed. It is the purpose of this note to show that with a proper choice of the dependent variables, the characteristic equations are much simpler, and the numerical integration can be carried out in a straightforward manner. ${ }^{2}$

2. The fundamental equations for one-dimensional unsteady flow are

$$
\begin{gathered}
u_{t}+u u_{x}+\frac{1}{\rho} p_{x}=0, \\
\rho_{t}+u \rho_{x}+\rho u_{x}+\rho u A^{-1} A_{x}=0, \\
s_{t}+u s_{x}=q^{*}, \\
p=\text { constant } \times \rho^{\gamma} \cdot e^{s},
\end{gathered}
$$

where $u$ is the velocity in the direction of the $x$-axis, $A$ is the cross-sectional area of the tube, $t$ is the time, and $p, \rho, s, \gamma$ are the familiar symbols for the pressure, the density, the entropy (divided by the specific heat at constant volume $c_{v}$ ) and the ratio of specific

*Received March 21, 1949.

${ }^{1}$ A. Kahane and L. Lees, J. Aero. Sci. 15, 665-670 (1948).

${ }^{2}$ For further discussions of this problem, see a note by William Swartz, which is to appear in J. Aero. Sci. 
heats, $q^{*}$ is the rate of production of entropy, i.e., it is the heat generated per unit mass divided by the product of absolute temperature $T$ and the specific heat at constant volume $c_{v}$.

The Eqs. (1)-(4) form a system of three differential equations in three variables, provided one of the four variables $p, \rho, s, u$ is eliminated by the use of (4). One can, e.g., eliminate the derivatives of $\rho$ from (2) to get

$$
p_{t}+u p_{x}+\gamma p u_{x}=p\left(q^{*}-\gamma u A^{-1} A_{x}\right) .
$$

The advantage in this choice lies in the fact that (1) and (2A) contain only the derivatives of the two variables $p$ and $u$.

3. The standard method of finding characteristics can now be applied. If we write

$$
\begin{aligned}
& p_{t} d t+p_{x} d x=d p \\
& u_{t} d t+u_{x} d x=d u \\
& s_{t} d t+s_{x} d x=d s
\end{aligned}
$$

and combine these equations with (1), (2A), and (3), we have six equations for the six partial derivatives. It can easily be seen that the coefficients and the right-hand sides of these equations can be arranged into the following matrix:

$$
\left(\begin{array}{lllllll}
1 & u & 0 & \rho^{-1} & 0 & 0 & 0 \\
0 & \gamma p & 1 & u & 0 & 0 & p\left(q^{*}-u \gamma A^{-1} A_{x}\right) \\
0 & 0 & 0 & 0 & 1 & u & q^{*} \\
d t & d x & 0 & 0 & 0 & 0 & d u \\
0 & 0 & d t & d x & 0 & 0 & d p \\
0 & 0 & 0 & 0 & d t & d x & d s
\end{array}\right)
$$

The coefficient determinant breaks down into two distinct factors

$$
d x-u d t=0, \quad(d x-u d t)^{2}-a^{2} d t^{2}=0 .
$$

As was expected, these are the familiar characteristic base curves. The corresponding compatibility equations can easily be obtained:

$$
\begin{gathered}
\frac{d s}{d t}=q^{*} \text { along } \frac{d x}{d t}=u \\
\frac{d p}{\gamma p} \pm \frac{d u}{a}=\left(\frac{q^{*}}{\gamma}-u A^{-1} A_{x}\right) d t \text { along } \frac{d x}{d t}=u \pm a .
\end{gathered}
$$

One may note in passing that these specialize immediately into those in the case of uniform entropy, provided the variables $p, u, s$ are also used in the latter case. In that case, with $A_{x}=0$, it is possible to integrate the compatibility equations (6). 
4. In the process of numerical integration, to reach a point $P_{3}$ from a knowledge of the solution along the curve $P_{1} P_{2}$ (Fig. 1) one may use the set of equations (6) to calculate $x_{3}, t_{3}, u_{3}$, and $p_{3}$, since $q^{*}$ and $A$ are supposed to be known functions. The

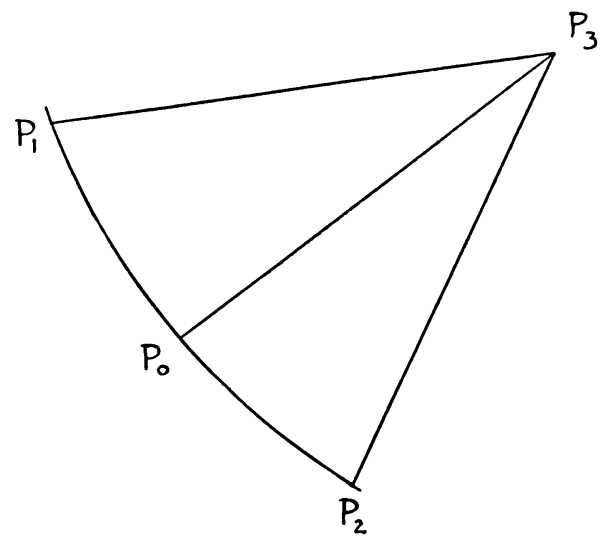

Fig. 1.

path line $P_{0} P_{3}$ can then be drawn in accordance with the second equation of (5); the first equation of (5) will give the value of $s_{3}$. One can then calculate $\rho_{3}$ from (4) and obtain all of the dynamic and thermodynamic variables at $P_{3}$. Iteration processes can be carried out in the usual manner.

\section{A NEW SUPERPOSITION PRINCIPLE FOR STEADY GAS FLOWS*}

\section{BY R. C. PRIM** (Naval Ordnance Laboratory)}

This paper is concerned with steady flows in the absence of extraneous fields of force of a frictionless, thermally-nonconducting gas having a product equation of state, i.e., an equation of state connecting density, pressure, and specific entropy in the form $\rho=P(p) S(s)$.

H. Poritsky [1] $\dagger$ has discussed the construction of steady, spatial gas flow solutions from steady plane flow solutions by the superposition of a uniform velocity field normal to the given plane flow field. In particular, he pointed out that if

$$
\mathbf{V}_{p}=\mathbf{i} u(x, y)+\mathbf{j} v(x, y)
$$

is a plane velocity field (referred to ordinary rectangular Cartesian coordinates $x, y, z$ with unit vectors $\mathbf{i}, \mathbf{j}, \mathbf{k}$ ) satisfying the equations of steady-state gas dynamics, then the spatial velocity field

$$
\mathrm{V}=\mathrm{V}_{p}+\mathbf{k} V_{n},
$$

where $V_{n}$ is a constant, also satisfies those equations.

*Received March 21, 1949.

**Now with Bell Telephone Laboratories.

$\dagger$ Numbers in square brackets refer to the bibliography at the end of the paper. 